\title{
PPARG C.1347C $>T$ polymorphism is associated with cancer susceptibility: from a case-control study to a meta-analysis
}

\author{
Hao Ding ${ }^{1, *}$, Yuanmei Chen ${ }^{2, *}$, Hao Qiu ${ }^{3, *}$, Chao Liu ${ }^{4}$, Yafeng Wang ${ }^{5}$, Mingqiang \\ Kang ${ }^{6}$ and Weifeng Tang 6 \\ ${ }^{1}$ Department of Respiratory Disease, Affiliated People's Hospital of Jiangsu University, Zhenjiang, Jiangsu Province, China \\ ${ }^{2}$ Department of Thoracic Surgery, Fujian Cancer Hospital, Fujian Medical University Cancer Hospital, Fuzhou, Fujian Province, \\ China \\ ${ }^{3}$ Department of Immunology, School of Medicine, Jiangsu University, Zhenjiang, Jiangsu Province, China \\ ${ }^{4}$ Department of Cardiothoracic Surgery, Affiliated People's Hospital of Jiangsu University, Zhenjiang, Jiangsu Province, China \\ ${ }^{5}$ Department of Cardiology, The People's Hospital of Xishuangbanna Dai Autonomous Prefecture, Jinghong, Yunnan Province, \\ China \\ ${ }^{6}$ Department of Thoracic Surgery, Fujian Medical University Union Hospital, Fuzhou, Fujian Province, China \\ *These authors contributed equally to this work
}

Correspondence to: Weifeng Tang, email: twf001001@126.com

Mingqiang Kang, email: Mingqiang_Kang @126.com

Keywords: PPARG, polymorphism, non-small cell lung cancer, risk

Received: July 03, 2017 Accepted: August 27, $2017 \quad$ Published: September 15, 2017

Copyright: Ding et al. This is an open-access article distributed under the terms of the Creative Commons Attribution License 3.0 (CC BY 3.0), which permits unrestricted use, distribution, and reproduction in any medium, provided the original author and source are credited.

\section{ABSTRACT}

Recently, several studies suggested that PPARG C.1347C $>T$ polymorphism was correlated with cancer risk. However, past results remained controversial. In this study, we performed a case-control study on the relationship of PPARG C.1347C>T polymorphism with risk of non-small cell lung cancer (NSCLC) and subsequently carried out a meta-analysis to further assess the association between PPARG C.1347C>T and overall cancer. In our case-control study, after adjusting by age, sex, body mass index (BMI), smoking and drinking, a tendency to increased NSCLC risk was noted (CT/TT vs. CC: adjusted OR, 1.21; 95\% CI, 0.97-1.51; $P=0.097)$. In the meta-analysis, we found a significant association between PPARG C.1347C $>T$ polymorphism and overall cancer risk (T vs. C: OR, 1.13; 95\% CI, 1.03-1.23; $P=0.006$; TT vs. CC: OR, $1.29 ; 95 \%$ CI, 1.07-1.56; $P=0.008, C T / T T$ vs. CC: $O R, 1.11 ; 95 \% C I, 1.02-1.21 ; P=0.014$ and TT vs. CT/CC: OR, 1.26; $95 \% C I, 1.04-1.52 ; P=0.016)$. In a subgroup analysis by ethnicity, evidence of significant association between PPARG C.1347C $>T$ polymorphism and cancer risk was found among Asians and mixed populations. In a subgroup analysis by cancer type, PPARG C.1347C $>T$ polymorphism was associated with risk of esophageal cancer and glioblastoma. In addition, in a subgroup analysis by origin of cancer cell, evidence of significant association between PPARG C.1347C $>T$ polymorphism and cancer risk was also found among epithelial tumor. In conclusion, the findings indicate PPARG c.1347C $>T$ polymorphism may increase the susceptibility of cancer.

\section{INTRODUCTION}

It is reported that about 14.1 million cancer patients and 8.2 million cancer-related deaths have occurred in 2012 worldwide [1]. In developing countries, the survival of cancer is poorer compared with the developed countries. The possible reason of this phenomenon is most likely due to limited access and lack of standard treatment. Cancer burden could be decreased through the application of tobacco control, healthier dietary intake, vaccine injection, early detection and treatment, and so on [2]. It is thought that cancer results from the interaction of individual's genetic components with environmental factors [3]. 
Peroxisome proliferator-activated receptor gamma (PPARG) involves three isoforms (e.g. PPARG1, PPARG2, and PPARG3). PPARG is an important nuclear receptor which acts as a transcriptional regulator and regulates energy metabolism [4]. In the pathological process of obesity, insulin insufficient/resistance and diabetes, PPARG may be activated, and then promotes the accumulation of fatty tissue [5]. PPARG agonists enhance insulin sensitivity [6]. PPARG may also possess anti-inflammatory roles $[7,8]$. Activation of PPARG could inhibit the production of many cytokines [e.g. tumor necrosis factor-alpha, interleukin (IL)-6, and IL8] by antagonizing the activities of the signal transducer and activator of transcription, transcription factors activator protein 1, and nuclear factor-kappa-B, which inhibits the induction of inflammatory response [9]. A number of case-control studies demonstrated that obesity, insulin resistance/insufficient, metabolic syndrome and inflammation were correlative conditions in which PPARG could modify and regulate these actions, and influence the risk of cancer [10-12].

Recently, a number of studies focused on the association of PPARG polymorphisms with cancer risk [13-28]. PPARG NM_015869.4:c.34C $>\mathrm{G}$ $($ rs1801282 C $>$ G) and NM_138712.3: c.1347C $>$ T (rs3856806 $\mathrm{C}>\mathrm{T}$ ) polymorphisms are two common single nucleotide polymorphisms (SNPs). A meta-analysis indicated the PPARG c.34C $>\mathrm{G}$ polymorphism was associated with the risk of cancer in Asians [29]. However, the association of PPARG c. $1347 \mathrm{C}>\mathrm{T}$ polymorphism with cancer risk was not found. Several meta-analyses did not identify the association between this SNP and cancer risk $[30,31]$. Although more and more case-control studies focused on the relationship of the PPARG c.1347C $>\mathrm{T}$ polymorphism with cancer susceptibility, the obtained findings remained conflicting. In addition, the association between this polymorphism and lung cancer was not studied in Asians. Therefore, in this study, we designed a case-control study and assessed the relationship between PPARG c. $1347 \mathrm{C}>\mathrm{T}$ polymorphism and risk of nonsmall cell lung cancer (NSCLC) in Eastern Chinese Han population. Meta-analysis is a useful method of promoting the effective sample size by pooling of individual data from the enrolled studies, thus strengthening the power of the study for the assessment of genetic effects [32]. To address the association between PPARG c. $1347 \mathrm{C}>\mathrm{T}$ polymorphism and cancer risk more precisely, we carried out a comprehensive meta-analysis.

\section{RESULTS}

\section{Association of PPARG c.1347C $>$ T polymorphism with NSCLC}

The risk factors, anthropometric data as well as demographics are listed in Table 1. Body mass index
(BMI) of controls was significantly higher than it in NSCLC group $(P<0.001)$. This study was well-matched by age and gender. The SNP information of PPARG c. $1347 \mathrm{C}>\mathrm{T}$ is shown in Table 2 . The genotyping success rate was $99.94 \%$ in 1,551 samples. Table 2 summarizes the minor allele frequency (MAF) of PPARG c.1347C $>\mathrm{T}$ polymorphism and Hardy-Weinberg Equilibrium (HWE) in controls.

The frequencies of PPARG c. $1347 \mathrm{CC}$, CT and TT genotypes were $57.01 \%, 38.00 \%$ and $4.99 \%$ in 521 NSCLC patients and $61.32 \%, 34.50 \%$, and $4.18 \%$ in 1,030 noncancer controls, respectively. The genotype distribution of PPARG c. $1347 \mathrm{C}>\mathrm{T}$ polymorphism is listed in Table 3 . In controls, the genotype distribution of this polymorphism was in accord with HWE. When compared with the frequency of c.1347 CC genotype, the frequency of c.1347 CT genotype was not difference between the NSCLC patients and controls (crude OR $=1.19,95 \%$ CI: 0.95-1.48, $P=0.130$ ). When compared with the frequency of c.1347 CC genotype, there was also no difference in the frequency of c.1347 TT genotype between the NSCLC patients and the controls (crude OR $=1.29,95 \% \mathrm{CI}$ : 0.78-2.13, $P=0.329$ ). When c. 1347 CC genotype was used as reference, there was also no difference in the frequency of c.1347 TT/CT genotype between the NSCLC patients and the controls (crude $\mathrm{OR}=1.20,95 \% \mathrm{CI}: 0.97-1.48, P=0.102)$. In addition, When c.1347 CC/CT genotype was used as reference, we found that there was no difference in the frequency of c.1347 TT genotype between the NSCLC patients and the controls (crude OR $=1.20,95 \% \mathrm{CI}$ : $0.73-1.98, P=0.465$ ). Adjustments for age, sex, BMI, smoking and drinking, as demonstrated in Table 3, a tendency to increased NSCLC risk was noted (CT vs. CC: adjusted OR, 1.21; 95\% CI, $0.96-1.53 ; P=0.106$; TT vs. CC: adjusted OR, $1.20 ; 95 \%$ CI, 0.71-2.04; $P=0.492, \mathrm{CT} / \mathrm{TT}$ vs. CC: adjusted OR, 1.21 ; 95\% CI, 0.97-1.51; $P=0.097$ and TT vs. CT/CC: adjusted OR, $1.12 ; 95 \%$ CI, 0.67-1.88; $P=0.671)$.

\section{Meta-analysis of PPARG c.1347C $>$ T polymorphism and cancer risk}

Next, we carried out a pooled analysis to determine the potential relationship between PPARG c.1347C $>\mathrm{T}$ polymorphism and overall cancer risk. A total of 35 abstracts were retrieved from searching of EMBASE and Pubmed databases. The selecting process of literature is presented in Figure 1. In total, there were 14 publications $[17,21,23,24,33-42]$ and our case-control study recruited in this meta-analysis. Some publications involved several subgroups [17, 21, 24, 33, 34, 38, 40, 42], we treated them separately. If 1 cancer type was studied by $<2$ individual studies, then it was combined into the subgroup of 'other cancers'. The characteristic of the included studies and PPARG c.1347C $>$ T genotypes in different study are listed in Tables 4, 5. In total, 6,814 cases and 14,590 controls were enrolled in this meta-analysis. 
Table 1: Distribution of selected demographic variables and risk factors in NSCLC cases and controls

\begin{tabular}{|c|c|c|c|}
\hline \multirow{2}{*}{ Variable } & Overall Cases $(n=521)$ & Overall Controls $(n=1,030)$ & \multirow{2}{*}{$P^{a}$} \\
\hline & $n(\%)$ & $n(\%)$ & \\
\hline Age (years) & $59.76 \pm 10.71$ & $60.34 \pm 9.11$ & 0.268 \\
\hline Age (years) & & & 0.843 \\
\hline$<60$ & $238(45.68)$ & $476(46.21)$ & \\
\hline$\geq 60$ & $283(54.32)$ & $554(53.79)$ & \\
\hline Sex & & & 0.453 \\
\hline Male & $287(55.09)$ & $588(57.09)$ & \\
\hline Female & $234(44.91)$ & $442(42.91)$ & \\
\hline Smoking status & & & $<0.001$ \\
\hline Never & $317(60.84)$ & $828(80.39)$ & \\
\hline Ever & $204(39.16)$ & $202(19.61)$ & \\
\hline Alcohol use & & & $<0.001$ \\
\hline Never & $444(85.22)$ & $949(92.14)$ & \\
\hline Ever & $77(14.78)$ & $81(7.86)$ & \\
\hline $\operatorname{BMI}\left(\mathrm{kg} / \mathrm{m}^{2}\right)$ & $23.00( \pm 3.03)$ & $23.84( \pm 3.06)$ & $<0.001$ \\
\hline \multicolumn{4}{|l|}{ BMI $\left(\mathrm{kg} / \mathrm{m}^{2}\right)$} \\
\hline$<24$ & $337(64.68)$ & $547(53.11)$ & $<0.001$ \\
\hline$\geq 24$ & $184(35.32)$ & $483(46.89)$ & \\
\hline
\end{tabular}

${ }^{a}$ Two-sided $\chi^{2}$ test and Student $t$ test

BMI: body mass index

Table 2: Primary information for PPARG c.1347C $>T$ polymorphism

\begin{tabular}{ll}
\hline Genotyped SNPs & PPARG c.1347C $>$ T \\
\hline Chromosome & 3 \\
Function & coding-synonymous \\
Chr Pos (NCBI Build 37) & 12475557 \\
MAFafor Chinese in database & 0.25 \\
MAF in our controls $(n=1,030)$ & 0.21 \\
$P$ value for HWE test in our controls & 0.431 \\
Genotyping method & SNPscan \\
\% Genotyping value & $99.94 \%$ \\
\hline
\end{tabular}

${ }^{a}$ MAF: minor allele frequency.

${ }^{b}$ HWE: Hardy-Weinberg equilibrium.

Overall, we found a significant association between PPARG c.1347C $>\mathrm{T}$ polymorphism and the increased risk of cancer (T vs. C: OR, 1.13; 95\% CI, 1.03-1.23; $P=0.006$; TT vs. CC: OR, $1.29 ; 95 \%$ CI, $1.07-1.56$; $P=0.008$, CT/TT vs. CC: OR, $1.11 ; 95 \%$ CI, $1.02-1.21$; $P=0.014$ and TT vs. CT/CC: OR, $1.26 ; 95 \%$ CI, 1.04 $1.52 ; P=0.016$; Table 6 and Figure 2).

In a subgroup analysis by the ethnicity, evidence of significant association between PPARG c.1347C $>\mathrm{T}$ polymorphism and increased risk of cancer were also found among Asians, and mixed populations, but not
Caucasians (Table 6). In a subgroup analysis by cancer type, c. $1347 \mathrm{C}>\mathrm{T}$ polymorphism was associated with the risk of esophageal cancer, and glioblastoma, but not biliary tract, breast, colorectal, melanoma, ovarian and other cancers (Table 6). In addition, in a subgroup analysis by the origin of cancer cell, evidence of significant association between $P P A R G$ c. $1347 \mathrm{C}>\mathrm{T}$ polymorphism and an increased risk of cancer were also found among epithelial tumor (Table 6).

The quality score of the enrolled studies was determined by using Newcastle-Ottawa Quality 
Table 3: Logistic regression analyses of associations between PPARG $c .1347 C>T$ polymorphism and risk of non-small cell lung cancer

\begin{tabular}{|c|c|c|c|c|c|c|c|c|}
\hline \multirow{2}{*}{ Genotype } & \multicolumn{2}{|c|}{ Cases $(n=521)$} & \multicolumn{2}{|c|}{ Controls $(n=1,030)$} & \multirow{2}{*}{$\begin{array}{c}\text { Crude OR } \\
(95 \% \mathrm{CI})\end{array}$} & \multirow{2}{*}{$P$} & \multirow{2}{*}{$\begin{array}{l}\text { Adjusted OR } \\
(95 \% \mathrm{a})\end{array}$} & \multirow{2}{*}{$\boldsymbol{P}$} \\
\hline & $n$ & $\%$ & $n$ & $\%$ & & & & \\
\hline \multicolumn{9}{|c|}{$P P A R \mathrm{G}$ c $.1347 \mathrm{C}>\mathrm{T}$} \\
\hline $\mathrm{CC}$ & 297 & 57.01 & 631 & 61.32 & 1.00 & & 1.00 & \\
\hline $\mathrm{CT}$ & 198 & 38.00 & 355 & 34.50 & $1.19(0.95-1.48)$ & 0.130 & $1.21(0.96-1.53)$ & 0.106 \\
\hline TT & 26 & 4.99 & 43 & 4.18 & $1.29(0.78-2.13)$ & 0.329 & $1.20(0.71-2.04)$ & 0.492 \\
\hline $\mathrm{CT}+\mathrm{TT}$ & 224 & 42.99 & 398 & 38.68 & $1.20(0.97-1.48)$ & 0.102 & $1.21(0.97-1.51)$ & 0.097 \\
\hline $\mathrm{CC}+\mathrm{CT}$ & 495 & 95.01 & 986 & 95.82 & 1.00 & & 1.00 & \\
\hline TT & 26 & 4.99 & 43 & 4.18 & $1.20(0.73-1.98)$ & 0.465 & $1.12(0.67-1.88)$ & 0.671 \\
\hline $\mathrm{T}$ allele & 250 & 23.99 & 441 & 21.43 & & & & \\
\hline
\end{tabular}

${ }^{a}$ Adjusted for age, sex, smoking status, alcohol use and BMI status.

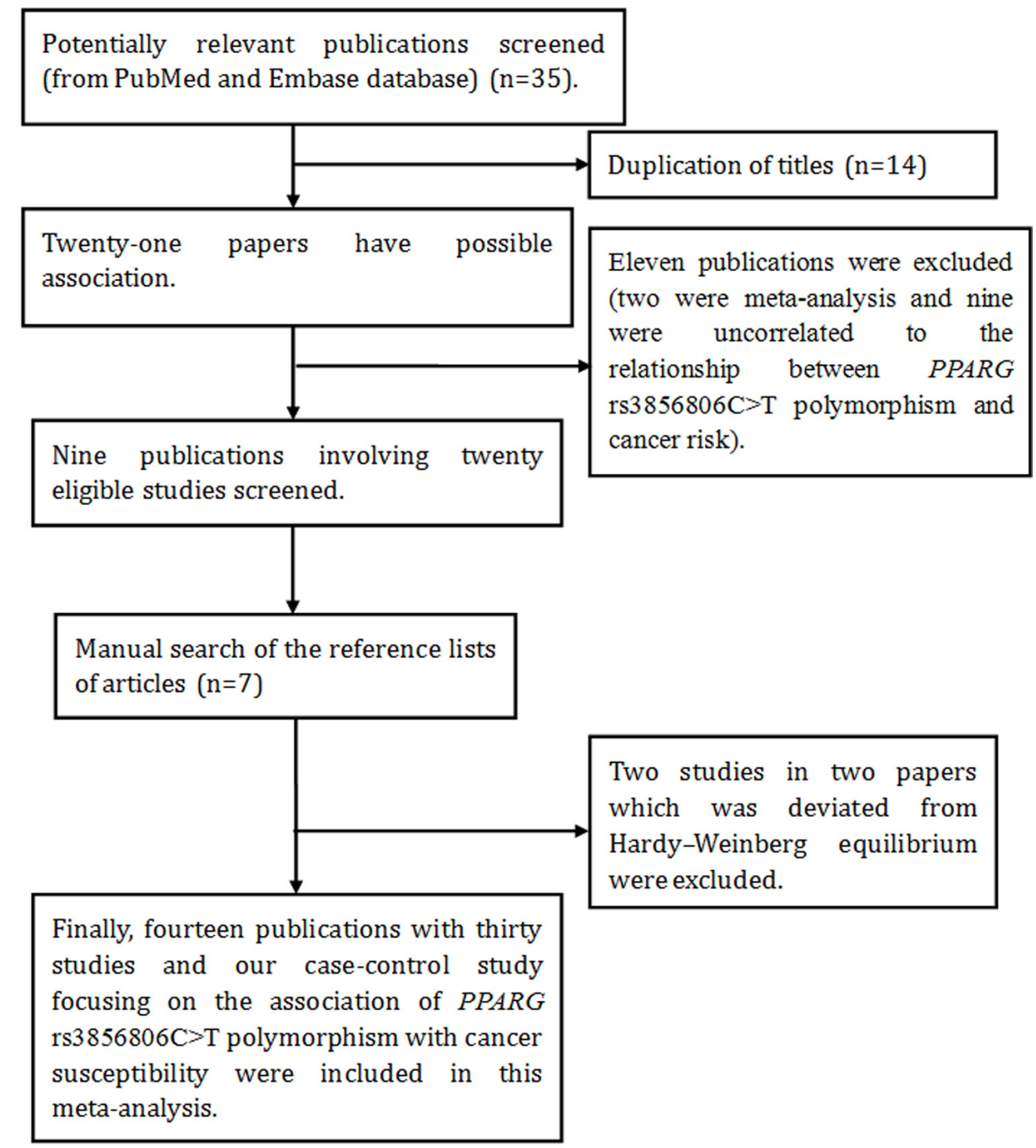

Figure 1: Flow diagram of the meta-analysis of the association between $P P A R G$ c.1347C $>$ T polymorphism and cancer risk. 
Table 4: Characteristics of the studies in meta-analysis

\begin{tabular}{|c|c|c|c|c|c|c|c|c|}
\hline Study & $\begin{array}{c}\text { Publication } \\
\text { year }\end{array}$ & Ethnicity & Country & Cancer type & $\begin{array}{c}\text { The origin of cancer } \\
\text { cell }\end{array}$ & $\begin{array}{l}\text { Sample size } \\
\text { (case/control) }\end{array}$ & Genotype method & Scores \\
\hline Zhou et al. [40] & 2000 & Caucasians & USA & Glioblastoma & Non-epithelial tumor & $52 / 80$ & DGGE & 4 \\
\hline Zhou et al. [40] & 2000 & Caucasians & German & Glioblastoma & Non-epithelial tumor & $44 / 60$ & DGGE & 4 \\
\hline Smith et al. [34] & 2001 & Asians & Japan & bladder cancer & Epithelial tumor & $31 / 27$ & DGGE & 2 \\
\hline Smith et al. [34] & 2001 & Asians & Japan & cervical cancer & Epithelial tumor & $20 / 27$ & DGGE & 2 \\
\hline Smith et al. [34] & 2001 & mixed & USA & endometrial cancer & Epithelial tumor & $69 / 80$ & DGGE & 2 \\
\hline Smith et al. [34] & 2001 & Caucasians & UK & ovarian cancer & Epithelial tumor & $31 / 65$ & DGGE & 2 \\
\hline Smith et al. [34] & 2001 & Asians & Japan & ovarian cancer & Epithelial tumor & $28 / 27$ & DGGE & 2 \\
\hline Smith et al. [34] & 2001 & mixed & USA & ovarian cancer & Epithelial tumor & $26 / 80$ & DGGE & 2 \\
\hline Smith et al. [34] & 2001 & mixed & USA & prostate cancer & Epithelial tumor & $38 / 80$ & DGGE & 2 \\
\hline Smith et al. [34] & 2001 & Caucasians & UK & Renal cell carcinoma & Epithelial tumor & $40 / 65$ & DGGE & 2 \\
\hline Jiang et al. [21] & 2005 & Asians & India & colorectal cancer & Epithelial tumor & $301 / 291$ & PCR-RFLP & 7 \\
\hline Jiang et al. [21] & 2005 & Asians & India & colorectal cancer & Epithelial tumor & $301 / 291$ & PCR-RFLP & 7 \\
\hline Siezen et al.[24] & 2006 & Caucasians & The netherlands & colorectal cancer & Epithelial tumor & $204 / 399$ & DNA sequence & 8 \\
\hline Siezen et al. [24] & 2006 & Caucasians & The netherlands & colorectal cancer & Epithelial tumor & $487 / 750$ & DNA sequence & 8 \\
\hline Kuriki et al. [17] & 2006 & Asians & Japanese & colorectal cancer & Epithelial tumor & $128 / 238$ & PCR-TCCP & 8 \\
\hline Kuriki et al. [17] & 2006 & Asians & Japanese & colorectal cancer & Epithelial tumor & $257 / 771$ & PCR-TCCP & 7 \\
\hline Wang et al. [41] & 2006 & mixed & USA & lymphoma & Non-epithelial tumor & $705 / 609$ & TaqMan & 8 \\
\hline Vogel et al. [23] & 2007 & Caucasians & Denmark & colorectal cancer & Epithelial tumor & $355 / 753$ & Not available & 8 \\
\hline Mossner et al.[42] & 2007 & Caucasians & German & melanoma & Non-epithelial tumor & $335 / 355$ & PCR-RFLP & 7 \\
\hline Mossner et al. [42] & 2007 & Caucasians & German & melanoma & Non-epithelial tumor & $497 / 435$ & PCR-RFLP & 7 \\
\hline Chang et al.[33] & 2008 & Asians & China & $\begin{array}{l}\text { ampulla of vater } \\
\text { cancer }\end{array}$ & Epithelial tumor & $47 / 786$ & TaqMan & 7 \\
\hline Chang et al. [33] & 2008 & Asians & China & bile duct cancer & Epithelial tumor & $127 / 786$ & TaqMan & 7 \\
\hline Doecke et al.[38] & 2008 & mixed & Australia & esophageal cancer & Epithelial tumor & $260 / 1352$ & sequencing & 7 \\
\hline Doecke et al. [38] & 2008 & mixed & Australia & esophageal cancer & Epithelial tumor & $301 / 1352$ & sequencing & 7 \\
\hline Doecke et al. [38] & 2008 & mixed & Australia & esophageal cancer & Epithelial tumor & $213 / 1352$ & sequencing & 7 \\
\hline Chang et al. [33] & 2008 & Asians & China & gallbladder cancer & Epithelial tumor & $237 / 786$ & TaqMan & 7 \\
\hline Wu et al. [35] & 2011 & Asians & China & breast cancer & Epithelial tumor & $291 / 589$ & RT-PCR & 7 \\
\hline Wei et al. [37] & 2013 & Asians & China & breast cancer & Epithelial tumor & $216 / 216$ & MALDI-TOF MS & 3 \\
\hline Jeon et al. [39] & 2013 & Asians & China & gastric cancer & Epithelial tumor & $196 / 397$ & TaqMan & 7 \\
\hline Park et al. [36] & 2014 & Asians & Korea & breast cancer & Epithelial tumor & $456 / 461$ & MALDI-TOF MS & 6 \\
\hline Our study & 2017 & Asians & China & lung cancer & Epithelial tumor & $521 / 1030$ & SNPscan & 7 \\
\hline
\end{tabular}

DGGE: denaturing gradient gel electrophoresis.

PCR-RFLP: polymerase chain reaction-restriction fragment length polymorphism.

PCR-CTPP: polymerase chain reaction with confronting two-pair primers.

RT-PCR: reverse transcription-polymerase chain reaction.

MALDI-TOF MS: Matrix-Assisted Laser Desorption/Ionization Time of Flight Mass Spectrometry.

Assessment Scale [43].The results indicated that nineteen were high-quality and twelve were low-quality (Table 7). When we excluded the low-quality studies, the results were not substantially altered suggesting the reliability of our findings (Table 6).
In this meta-anlysis, we used Begg's test and Egger's test to measure the publication bias. The results demonstrated that there was no significant bias in any genetic model (T vs. C: Begg's test $P=0.442$, Egger's test $P=0.196$; TT vs. CC: Begg's test $P=0.442$, Egger's 
Table 5: Distribution of $P P A R G$ c.1347C $>$ T polymorphism genotype and allele

\begin{tabular}{|c|c|c|c|c|c|c|c|c|c|c|c|c|}
\hline \multirow{2}{*}{ Study } & \multirow{2}{*}{$\begin{array}{c}\text { Publication } \\
\text { year }\end{array}$} & \multicolumn{3}{|c|}{ case } & \multicolumn{3}{|c|}{ control } & \multicolumn{2}{|c|}{ case } & \multicolumn{2}{|c|}{ contraol } & \multirow{2}{*}{ HWE } \\
\hline & & $\mathrm{CC}$ & CT & TT & $\mathrm{CC}$ & CT & TT & $\mathbf{T}$ & $\mathbf{C}$ & $\mathbf{T}$ & $\mathbf{C}$ & \\
\hline Zhou et al. [40] & 2000 & 31 & 21 & 0 & 70 & 10 & 0 & 21 & 83 & 10 & 150 & Yes \\
\hline Zhou et al. [40] & 2000 & 33 & 10 & 1 & 49 & 11 & 0 & 12 & 76 & 11 & 109 & Yes \\
\hline Smith et al. [34] & 2001 & 27 & 7 & 0 & 18 & 9 & 0 & 7 & 61 & 9 & 45 & Yes \\
\hline Smith et al. [34] & 2001 & 17 & 3 & 0 & 18 & 9 & 0 & 3 & 37 & 9 & 45 & Yes \\
\hline Smith et al. [34] & 2001 & 53 & 12 & 4 & 70 & 10 & 0 & 20 & 118 & 10 & 150 & Yes \\
\hline Smith et al. [34] & 2001 & 27 & 4 & 0 & 52 & 12 & 1 & 4 & 58 & 14 & 116 & Yes \\
\hline Smith et al. [34] & 2001 & 19 & 9 & 0 & 18 & 9 & 0 & 9 & 47 & 9 & 45 & Yes \\
\hline Smith et al. [34] & 2001 & 20 & 6 & 0 & 70 & 10 & 0 & 6 & 46 & 10 & 150 & Yes \\
\hline Smith et al. [34] & 2001 & 30 & 6 & 2 & 70 & 10 & 0 & 10 & 66 & 10 & 150 & Yes \\
\hline Smith et al. [34] & 2001 & 29 & 11 & 0 & 52 & 12 & 1 & 11 & 69 & 14 & 116 & Yes \\
\hline Jiang et al. [21] & 2005 & 37 & 19 & 3 & 221 & 66 & 4 & 25 & 93 & 74 & 508 & Yes \\
\hline Jiang et al. [21] & 2005 & 173 & 61 & 8 & 221 & 66 & 4 & 77 & 407 & 74 & 508 & Yes \\
\hline Siezen et al. [24] & 2006 & 155 & 42 & 4 & 307 & 79 & 4 & 50 & 352 & 87 & 693 & Yes \\
\hline Siezen et al. [24] & 2006 & 380 & 92 & 7 & 555 & 162 & 9 & 106 & 852 & 180 & 1272 & Yes \\
\hline Kuriki et al. .[17] & 2006 & 92 & & $35^{*}$ & 117 & & $61^{*}$ & & & & & Yes \\
\hline Kuriki et al. .[17] & 2006 & 184 & & $73^{*}$ & 543 & & $226^{*}$ & & & & & Yes \\
\hline Wang et al. .[41] & 2006 & 537 & 150 & 18 & 459 & 137 & 13 & 186 & 1224 & 163 & 1055 & Yes \\
\hline Vogel et al. [23] & 2007 & 255 & 96 & 4 & 557 & 181 & 15 & 104 & 606 & 211 & 1295 & Yes \\
\hline Mossner et al. [42] & 2007 & 242 & 73 & 20 & 273 & 73 & 7 & 113 & 557 & 87 & 619 & Yes \\
\hline Mossner et al. [42] & 2007 & 377 & 113 & 7 & 316 & 111 & 8 & 127 & 867 & 127 & 743 & Yes \\
\hline Chang et al. [33] & 2008 & 27 & 18 & 2 & 457 & 284 & 41 & 22 & 72 & 366 & 1198 & Yes \\
\hline Chang et al. [33] & 2008 & 74 & 44 & 8 & 457 & 284 & 41 & 60 & 192 & 366 & 1198 & Yes \\
\hline Doecke et al. [38] & 2008 & 190 & 65 & 5 & 1068 & 270 & 14 & 75 & 445 & 298 & 2406 & Yes \\
\hline Doecke et al. [38] & 2008 & 223 & 72 & 6 & 1068 & 270 & 14 & 84 & 518 & 298 & 2406 & Yes \\
\hline Doecke et al. [38] & 2008 & 170 & 41 & 2 & 1068 & 270 & 14 & 45 & 381 & 298 & 2406 & Yes \\
\hline Chang et al. [33] & 2008 & 127 & 95 & 15 & 457 & 284 & 41 & 125 & 349 & 366 & 1198 & Yes \\
\hline Wu et al. [35] & 2011 & 162 & 110 & 19 & 328 & 219 & 40 & 148 & 434 & 299 & 875 & Yes \\
\hline Wei et al. [37] & 2013 & 115 & 69 & 15 & 122 & 69 & 9 & 99 & 299 & 87 & 313 & Yes \\
\hline Jeon et al. .[39] & 2013 & 104 & 75 & 12 & 220 & 141 & 22 & 99 & 283 & 185 & 581 & Yes \\
\hline Park et al. [36] & 2014 & 320 & 126 & 8 & 311 & 117 & 15 & 142 & 766 & 147 & 739 & Yes \\
\hline Our study & 2017 & 297 & 198 & 26 & 631 & 355 & 43 & 250 & 792 & 441 & 1617 & Yes \\
\hline
\end{tabular}

"Indicates TT $+\mathrm{CT}$

HWE: Hardy-Weinberg equilibrium.

test $P=0.167 ; \mathrm{CT} / \mathrm{TT}$ vs. CC: Begg's test $P=0.634$, Egger's test $P=0.244$; TT vs. CT/CC: Begg's test $P=$ 0.333 , Egger's test $P=0.149$; Figure 3 ). Using the oneway method (excluding an individual study in turn), sensitivity analysis was carried out to determine stability of our findings (Figure 4). The results indicated that our findings were stable and reliable.

Significant heterogeneities were found in this metaanalysis. Since the origin of cancer cell, quality score, ethnicity and cancer type could affect the results of meta- analysis, we carried out subgroup analyses by these factors and the findings were presented in Table 6. The results indicated that melanoma, non-epithelial tumor, Caucasians and quality score $<7.0$ subgroups may contribute to the major heterogeneity. As shown in Table 6, significant heterogeneity was found in allele comparison, thus meta-regression was also performed to explore the source of heterogeneity. We found that quality score might contributed to major heterogeneity, which can explain $64.27 \%$ heterogeneity (Tau1 $=0.019100$, Tau $2=0.006824$, respectively). 


\section{DISCUSSION}

The etiology of cancer was very complex. It is thought that many environmental and genetic factors may play important roles in the development of cancer. Multiple lines of evidence indicate a vital role for genetics in determining risk for cancer. PPARG is a member of the peroxisome proliferator-activated receptors (PPARs). PPARs interact with retinoid $\mathrm{X}$ receptors and then regulate the transcription process of many genes. PPARG has been implicated in the development of various diseases involving obesity, diabetes, inflammation, atherosclerosis and cancer [44-47]. PPARG is expressed in various cancer cells. There are accumulating evidences that obesity/ overweight, type 2 diabetes, inflammation, and malignancy are etiologically related $[48,49]$. Being at the crossroads of multiple diseases, PPARG may be a key component for understanding the pathophysiology of cancer. In this study, we explored the relationship of $P P A R G$ c. $1347 \mathrm{C}>\mathrm{T}$ polymorphism with NSCLC risk. Then, we conducted a comprehensive meta-analysis to further understand the potential role of this SNP for the susceptibility to overall cancer. In the case-control study, we found an association between PPARG c.1347C $>\mathrm{T}$ polymorphism and a tendency to increased risk of NSCLC. Along with a metaanalysis, we found that $P P A R G \mathrm{c} .1347 \mathrm{C}>\mathrm{T}$ polymorphism was associated with the increased risk of overall cancer. To the best of our knowledge, this study is the first casecontrol study focusing on the association between PPARG c. $1347 \mathrm{C}>\mathrm{T}$ polymorphism and NSCLC risk in Asians. And we first confirmed the relationship between this SNP and overall cancer risk.

With the increasing studies on genetic association, it is necessary to analyze the available data to obtain robust, replicable results. Considering the fact that a common SNP may make a small-to-moderate contribution to the risk of

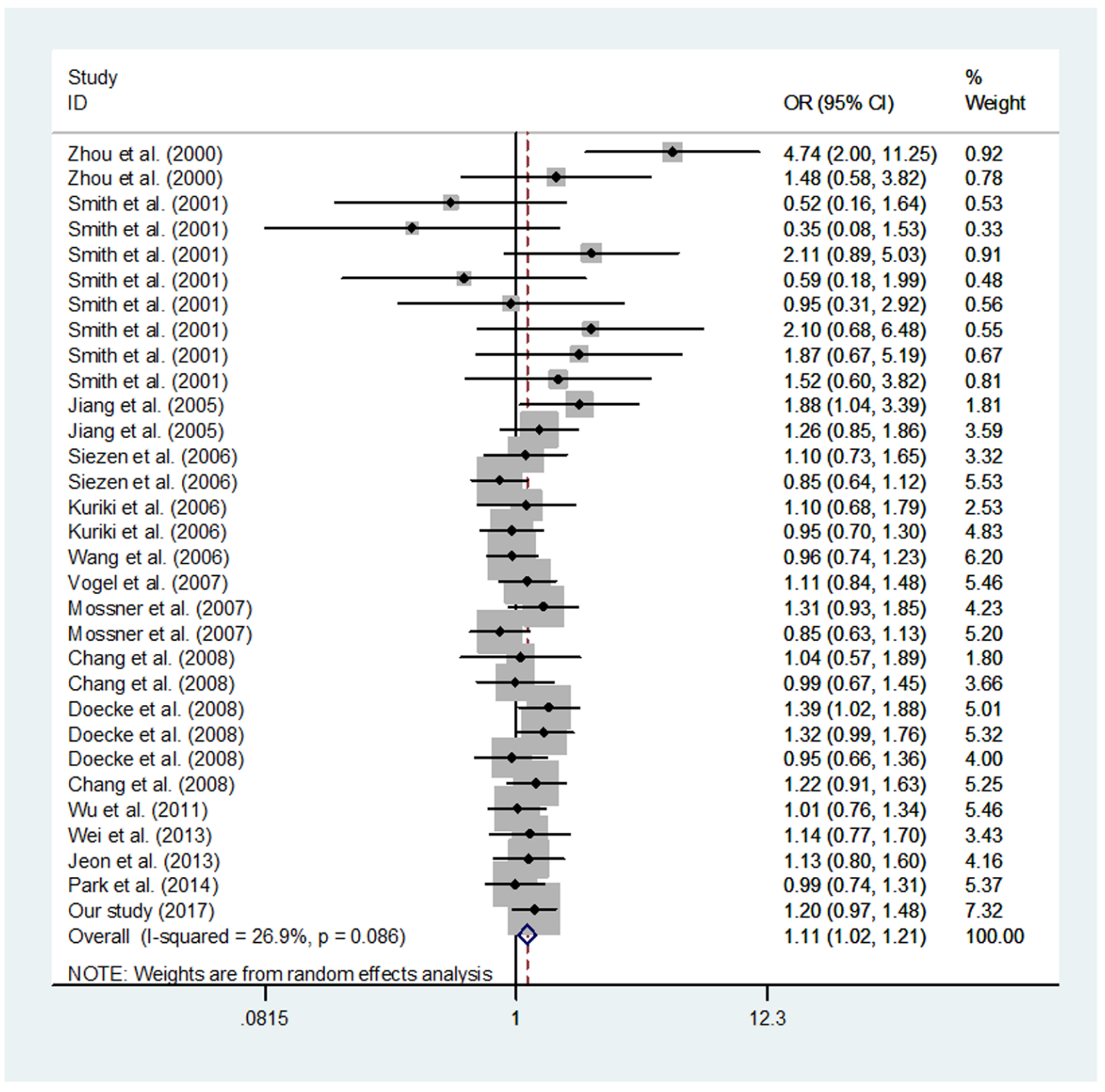

Figure 2: Meta-analysis of the association between PPARG c.1347C $>$ T polymorphism and cancer risk (TT/CT vs. CC, random-effects model). 
Table 6: Results of the meta-analysis from different comparative genetic models

\begin{tabular}{|c|c|c|c|c|c|c|c|c|c|c|c|c|c|c|c|c|c|}
\hline & \multirow{2}{*}{$\begin{array}{l}\text { No. of } \\
\text { studies }\end{array}$} & \multicolumn{4}{|c|}{ T vs. C } & \multicolumn{4}{|c|}{ TT vs. CC } & \multicolumn{4}{|c|}{$\mathrm{TT}+\mathrm{CT}$ vs. CC } & \multicolumn{4}{|c|}{ TT vs. $\mathrm{CT}+\mathrm{CC}$} \\
\hline & & OR $(95 \% \mathrm{CI})$ & $P$ & 12 & $\begin{array}{l}\mathrm{P}(\mathbf{Q}- \\
\text { test) }\end{array}$ & OR(95\% CI) & $\mathbf{P}$ & 12 & P(Q-test) & OR $(95 \% \mathrm{CI})$ & $P$ & 12 & $\mathbf{P}(\mathbf{Q}$-test) & $\mathrm{OR}(95 \% \mathrm{CI})$ & $P$ & 12 & $\begin{array}{l}\mathbf{P}(\mathbf{Q}- \\
\text { test) }\end{array}$ \\
\hline Total & 31 & $1.13(1.03-1.23)$ & 0.006 & $39.5 \%$ & 0.016 & $1.29(1.07-1.56)$ & 0.008 & $6.6 \%$ & 0.370 & $1.11(1.02-1.21)$ & 0.014 & $26.9 \%$ & 0.086 & $1.26(1.04-1.52)$ & 0.016 & $1.9 \%$ & 0.436 \\
\hline \multicolumn{18}{|l|}{ Ethnicity } \\
\hline Asians & 15 & $1.10(1.01-1.20)$ & 0.033 & $0.1 \%$ & 0.444 & $1.19(0.94-1.51)$ & 0.149 & $6.4 \%$ & 0.382 & $1.10(1.00-1.21)$ & 0.058 & $0.0 \%$ & 0.704 & $1.15(0.91-1.45)$ & 0.248 & $0.0 \%$ & 0.447 \\
\hline Caucasians & 9 & $1.14(0.91-1.43)$ & 0.246 & $61.1 \%$ & 0.008 & $1.33(0.87-2.03)$ & 0.192 & $19.5 \%$ & 0.275 & $1.14(0.89-1.45)$ & 0.290 & $59.8 \%$ & 0.011 & $1.32(0.87-2.02)$ & 0.196 & $17.4 \%$ & 0.293 \\
\hline Mixed & 7 & $1.26(1.02-1.55)$ & 0.034 & $50.5 \%$ & 0.059 & $1.70(1.08-2.68)$ & 0.022 & $1.7 \%$ & 0.405 & $1.17(1.01-1.35)$ & 0.032 & 34.3 & 0.166 & $1.67(1.06-2.63)$ & 0.027 & $0.0 \%$ & 0.462 \\
\hline \multicolumn{18}{|l|}{ Cancer type } \\
\hline $\begin{array}{l}\text { Biliary tract } \\
\text { cancer }\end{array}$ & 3 & $1.10(0.92-1.31)$ & 0.288 & $0.0 \%$ & 0.729 & $1.21(0.76-1.93)$ & 0.416 & $0.0 \%$ & 0.848 & $1.12(0.90-1.38)$ & 0.322 & $0.0 \%$ & 0.675 & $1.17(0.74-1.84)$ & 0.508 & $0.0 \%$ & 0.865 \\
\hline Breast cancer & 3 & $1.01(0.87-1.17)$ & 0.890 & $0.0 \%$ & 0.504 & $0.96(0.63-1.45)$ & 0.843 & $47.8 \%$ & 0.147 & $1.03(0.86-1.23)$ & 0.785 & $0.0 \%$ & 0.834 & $0.95(0.63-1.43)$ & 0.810 & $48.1 \%$ & 0.145 \\
\hline Colorectal cancer & 7 & $1.10(0.97-1.25)$ & 0.148 & $50.1 \%$ & 0.143 & $1.48(0.95-2.30)$ & 0.084 & $32.8 \%$ & 0.299 & $1.06(0.94-1.21)$ & 0.353 & $19.6 \%$ & 0.370 & $1.45(0.93-2.25)$ & 0.099 & $27.2 \%$ & 0.345 \\
\hline Esophageal cancer & 3 & $1.22(1.04-1.44)$ & 0.016 & $33.7 \%$ & 0.221 & $1.69(0.90-3.18)$ & 0.102 & $0.0 \%$ & 0.621 & $1.23(1.03-1.47)$ & 0.025 & $27.7 \%$ & 0.251 & $1.62(0.86-3.05)$ & 0.131 & $0.0 \%$ & 0.671 \\
\hline Glioblastoma & 2 & $2.54(1.43-4.54)$ & 0.002 & $53.8 \%$ & 0.141 & - & - & - & - & $2.70(0.86-8.41)$ & 0.087 & $68.4 \%$ & 0.075 & - & - & - & - \\
\hline Melanoma & 2 & $1.11(0.66-1.84)$ & 0.698 & $84.5 \%$ & 0.011 & $1.58(0.37-6.73)$ & 0.539 & $78.4 \%$ & 0.031 & $1.04(0.68-1.60)$ & 0.849 & $72.2 \%$ & 0.058 & $1.59(0.40-6.36)$ & 0.514 & $76.5 \%$ & 0.039 \\
\hline Ovarian cancer & 3 & $1.01(0.55-1.86)$ & 0.975 & $17.6 \%$ & 0.297 & - & - & - & - & $1.05(0.54-2.02)$ & 0.887 & $14.5 \%$ & 0.311 & - & - & - & - \\
\hline Other cancers & 8 & $1.11(0.99-1.25)$ & 0.085 & $39.1 \%$ & 0.118 & $1.35(0.96-1.91)$ & 0.088 & $0.0 \%$ & 0.471 & $1.11(0.96-1.28)$ & 0.148 & $25.2 \%$ & 0.228 & $1.29(0.92-1.82)$ & 0.141 & $0.0 \%$ & 0.476 \\
\hline \multicolumn{18}{|l|}{$\begin{array}{l}\text { The origin of } \\
\text { cancer cell }\end{array}$} \\
\hline Epithelial tumor & 26 & $1.11(1.04-1.19)$ & 0.003 & $20.5 \%$ & 0.183 & $1.25(1.01-1.54)$ & 0.036 & $0.0 \%$ & 0.483 & $1.11(1.03-1.20)$ & 0.007 & $0.0 \%$ & 0.500 & $1.21(0.98-1.48)$ & 0.074 & $0.0 \%$ & 0.547 \\
\hline $\begin{array}{l}\text { Non-epithelial } \\
\text { tumor }\end{array}$ & 5 & $1.29(0.91-1.83)$ & 0.161 & $76.9 \%$ & 0.002 & $1.54(0.96-2.46)$ & 0.073 & $46.9 \%$ & 0.130 & $1.27(0.87-1.86)$ & 0.220 & $75.6 \%$ & 0.003 & $1.55(0.97-2.47)$ & 0.069 & $42.2 \%$ & 0.158 \\
\hline \multicolumn{18}{|l|}{ Quality scores } \\
\hline$\geq 7.0$ & 19 & $1.01(1.03-1.18)$ & 0.005 & $27.0 \%$ & 0.146 & $1.30(1.06-1.59)$ & 0.012 & $0.0 \%$ & 0.540 & $1.09(1.01-1.17)$ & 0.021 & $5.7 \%$ & 0.386 & $1.26(1.03-1.54)$ & 0.025 & $0.0 \%$ & 0.614 \\
\hline$<7.0$ & 12 & $1.30(0.95-1.77)$ & 0.099 & $54.4 \%$ & 0.012 & $1.28(0.77-2.14)$ & 0.348 & $38.8 \%$ & 0.133 & $1.29(0.93-1.78)$ & 0.129 & $48.7 \%$ & 0.029 & $1.25(0.75-2.08)$ & 0.388 & $37.7 \%$ & 0.141 \\
\hline
\end{tabular}

cancer, this pooled-analysis urges the necessity of adequate sample sizes to get a precise measurement between $P P A R G$ c. $1347 \mathrm{C}>\mathrm{T}$ polymorphism and the development of cancer. Several individual studies have reported positive signals of PPARG c. $1347 \mathrm{C}>\mathrm{T}$ polymorphism with cancer risk
$[21,38,40]$; however, others observed null association. Recently, a meta-analysis reported that this polymorphism was not associated with cancer risk [31]; however, this pooled-analysis only included four case-control studies. In this updated meta-analysis, overall findings among 21,404

\section{Begg's funnel plot with pseudo $95 \%$ confidence limits}

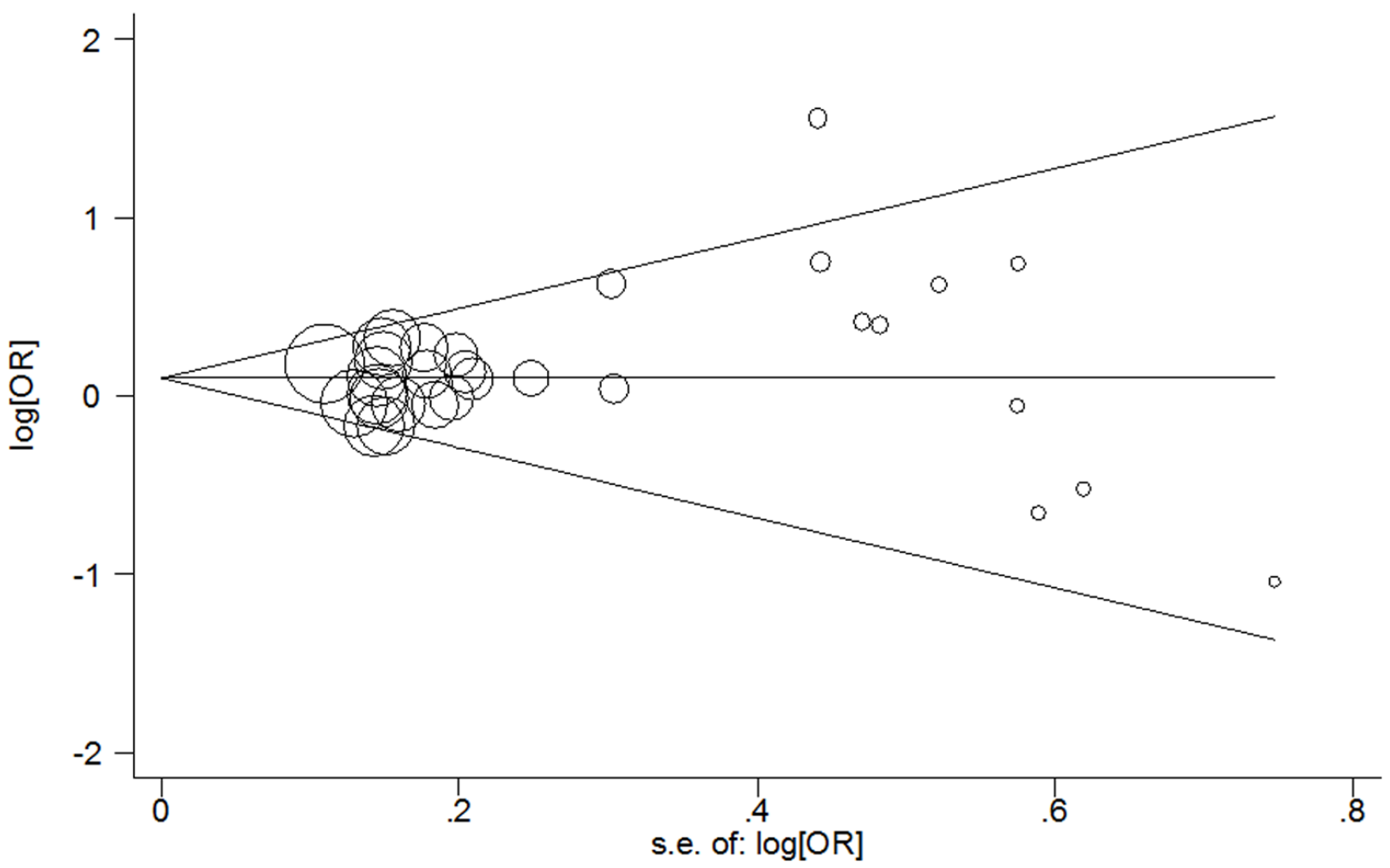

Figure 3: Begg's funnel plot of meta-analysis of the association between $P P A R G$ c.1347C $>$ T polymorphism and cancer risk (TT/CT vs. CC compare genetic model, random-effects model). 


\begin{tabular}{|c|c|c|c|c|c|c|c|c|c|c|}
\hline \multirow[b]{2}{*}{ Study } & \multirow[b]{2}{*}{ Year } & \multicolumn{4}{|c|}{ Selection } & \multirow{2}{*}{$\begin{array}{l}\text { Comparability } \\
\text { of the cases and } \\
\text { controls }\end{array}$} & \multicolumn{3}{|c|}{ Exposure } & \multirow[b]{2}{*}{$\begin{array}{l}\text { Total } \\
\text { Stars }\end{array}$} \\
\hline & & $\begin{array}{l}\text { Adequate case } \\
\text { definition }\end{array}$ & $\begin{array}{l}\text { Representativeness } \\
\text { of the cases }\end{array}$ & $\begin{array}{l}\text { Selection of } \\
\text { the controls }\end{array}$ & $\begin{array}{l}\text { Definition of } \\
\text { Controls }\end{array}$ & & $\begin{array}{l}\text { Ascertainment } \\
\text { of exposure }\end{array}$ & $\begin{array}{c}\text { Same } \\
\text { ascertainment } \\
\text { method for cases } \\
\text { and controls }\end{array}$ & $\begin{array}{c}\text { Non- } \\
\text { Response rate }\end{array}$ & \\
\hline Zhou et al. [40] & 2000 & $\star$ & $\star$ & - & $\star$ & - & $\star$ & - & - & 4 \\
\hline Zhou et al. [40] & 2001 & $\star$ & $\star$ & - & $\star$ & - & $\star$ & - & - & 4 \\
\hline Smith et al. [34] & 2001 & - & - & - & $\star$ & $\star$ & - & - & - & 2 \\
\hline Smith et al. [34] & 2001 & - & - & - & $\star$ & $\star$ & - & - & - & 2 \\
\hline Smith et al. [34] & 2001 & - & - & - & $\star$ & $\star$ & - & - & - & 2 \\
\hline Smith et al. [34] & 2001 & - & - & - & $\star$ & $\star$ & - & - & - & 2 \\
\hline Smith et al. [34] & 2001 & - & - & - & $\star$ & $\star$ & - & - & - & 2 \\
\hline Smith et al. [34] & 2001 & - & - & - & $\star$ & $\star$ & - & - & - & 2 \\
\hline Smith et al. [34] & 2001 & - & - & - & $\star$ & $\star$ & - & - & - & 2 \\
\hline Smith et al. [34] & 2001 & - & - & - & $\star$ & $\star$ & - & - & - & 2 \\
\hline Jiang et al. [21] & 2005 & $\star$ & $\star$ & - & $\star$ & $\star \star$ & $\star$ & $\star$ & - & 7 \\
\hline Jiang et al. [21] & 2005 & $\star$ & $\star$ & - & $\star$ & $\star \star$ & $\star$ & $\star$ & - & 7 \\
\hline Siezen et al. [24] & 2006 & $\star$ & $\star$ & $\star$ & $\star$ & $\star \star$ & $\star$ & $\star$ & - & 8 \\
\hline Siezen et al. [24] & 2006 & $\star$ & $\star$ & $\star$ & $\star$ & $\star \star$ & $\star$ & $\star$ & - & 8 \\
\hline Kurikin et al. [17] & 2006 & $\star$ & $\star$ & - & $\star$ & $\star \star$ & $\star$ & $\star$ & $\star$ & 8 \\
\hline Kurikin et al. [17] & 2006 & $\star$ & $\star$ & - & $\star$ & $\star \star$ & $\star$ & $\star$ & - & 7 \\
\hline Wang et al. [41] & 2006 & $\star$ & $\star$ & $\star$ & $\star$ & $\star \star$ & $\star$ & - & $\star$ & 8 \\
\hline Vogel et al. [23] & 2007 & $\star$ & $\star$ & $\star$ & $\star$ & $\star \star$ & $\star$ & $\star$ & - & 8 \\
\hline Mossner et al. [42] & 2007 & $\star$ & $\star$ & - & $\star$ & $\star \star$ & $\star$ & $\star$ & - & 7 \\
\hline Mossner et al. [42] & 2007 & $\star$ & $\star$ & - & $\star$ & $\star \star$ & $\star$ & $\star$ & - & 7 \\
\hline Chang et al. [39 & 2008 & $\star$ & $\star$ & - & $\star$ & $\star \star$ & $\star$ & $\star$ & - & 7 \\
\hline Chang et al. [39 & 2008 & $\star$ & $\star$ & - & $\star$ & $\star \star$ & $\star$ & $\star$ & - & 7 \\
\hline Chang et al. [39 & 2008 & $\star$ & $\star$ & - & $\star$ & $\star \star$ & $\star$ & $\star$ & - & 7 \\
\hline Doecke et al. [38] & 2008 & $\star$ & $\star$ & $\star$ & $\star$ & $\star \star$ & $\star$ & - & - & 7 \\
\hline Doecke et al. [38] & 2008 & $\star$ & $\star$ & $\star$ & $\star$ & $\star \star$ & $\star$ & - & - & 7 \\
\hline Doecke et al. [38] & 2008 & $\star$ & $\star$ & $\star$ & $\star$ & $\star \star$ & $\star$ & - & - & 7 \\
\hline Wu et al. [35] & 2011 & $\star$ & $\star$ & - & $\star$ & $\star \star$ & $\star$ & $\star$ & - & 7 \\
\hline Wei et al. [37] & 2013 & $\star$ & - & - & $\star$ & - & $\star$ & - & - & 3 \\
\hline Jeon et al. [39] & 2013 & - & $\star$ & $\star$ & $\star$ & $\star \star$ & $\star$ & $\star$ & - & 7 \\
\hline Park et al. [36] & 2014 & $\star$ & $\star$ & - & - & $\star \star$ & $\star$ & $\star$ & - & 6 \\
\hline Our study & 2017 & $\star$ & $\star$ & - & $\star$ & $\star \star$ & $\star$ & $\star$ & - & 7 \\
\hline
\end{tabular}

subjects, evidence of significant association between this polymorphism and cancer risk were found, even in Asians, mixed populations, esophageal cancer, glioblastoma and epithelial tumor subgroups. In PPARG exon 6, a C to $\mathrm{T}$ substitution is a synonymous polymorphism which encodes histidine either with PPARG c.1347 C or T allele. The findings of previous epidemiological studies showed a relationship of this polymorphism with metabolic diseases such as type 2 diabetes and atherosclerosis [50-53]. It is proposed that the $\mathrm{C}$ to $\mathrm{T}$ substitution may modulate the expression of PPARG by altering mRNA processing or translation. A tendency of increased risk was observed for PPARG c.1347C $>$ T polymorphism with
NSCLC risk, and an increased risk was also found in the subsequent meta-analysis. These consistent findings demonstrated that $P P A R G$ c. $1347 \mathrm{C}>\mathrm{TC}>\mathrm{T}$ polymorphism might influence the development of cancer. In the future, further evaluations with detailed environmental factors are warranted to confirm these results.

Additionally, some potential limitations should be further addressed when interpreting our findings. First, the design of our case-control study was hospital-based, and the selecting bias might have occurred. Second, in this meta-analysis, the included studies based on the published studies, unpublished articles might fail to be retrieved. Third, since the significant heterogeneities were found 
in this meta-analysis, our findings should be interpreted with cautions. Fourth, lack of the data on environmental factors (e.g. lifestyle, fasting plasma glucose, total cholesterol, high-density lipoprotein cholesterol, lowdensity lipoprotein cholesterol, serum triglycerides etc.), the corresponding subgroup analyses were not conducted. Finally, we only focused on c. $1347 \mathrm{C}>\mathrm{T}$ polymorphism in PPARG gene, and did not consider other susceptibility genes or polymorphisms.

In conclusion, this case-control study in Eastern Chinese Han populations, along with a comprehensive meta-analysis, identify the association of PPARG c. $1347 \mathrm{C}>\mathrm{T}$ polymorphism with an increased risk of cancer, even in Asians, esophageal cancer, glioblastoma and epithelial tumor subgroups. Nevertheless, for some practical reasons, we hope that more case-control studies with the detailed environmental data to further explore the molecular mechanism of PPARG c. $1347 \mathrm{C}>\mathrm{T}$ polymorphism with development of cancer.

\section{MATERIALS AND METHODS}

\section{Subjects}

Genotyping analyses were carried out on genomic DNA of 521 NSCLC patients and 1,030 unrelated controls. All participants were come from Eastern Chinese Han population. The major included criterion of NSCLC patients were: (A) living in Eastern China area; (B) NSCLC was confirmed by pathological examination; (C) without autoimmune disease. The NSCLC patients comprised unrelated subjects who had been treated in Affiliated People's Hospital of Jiangsu University and Fujian Medical University Union Hospital. The blood samples were collected from January 2014 to December 2016. Index cases were first diagnosed with NSCLC. All patients gave a written informed consent.

The controls included healthy blood donors collected in the same hospitals, having the same ethnic background and similar lifestyle as the NSCLC patients. The controls were biologically unrelated to the NSCLC cases and were cancer-free. The age distribution of NSCLC cases and non-cancer controls was nearly identical (controls: $60.34 \pm 9.11$ years; cases: $59.76 \pm 10.71$ years; $P=0.268)$. The sex distribution of NSCLC cases and controls was well-matched $(P=0.453)$. According to the guidelines of Chinese blood donation, each participant was examined by a questionnaire and wrote his/her informed consent. The controls were randomly collected during the years 20142016. The study was approved by the Ethics Committee of Jiangsu University (Zhenjiang, China) and Fujian Medical University (Fuzhou, China).

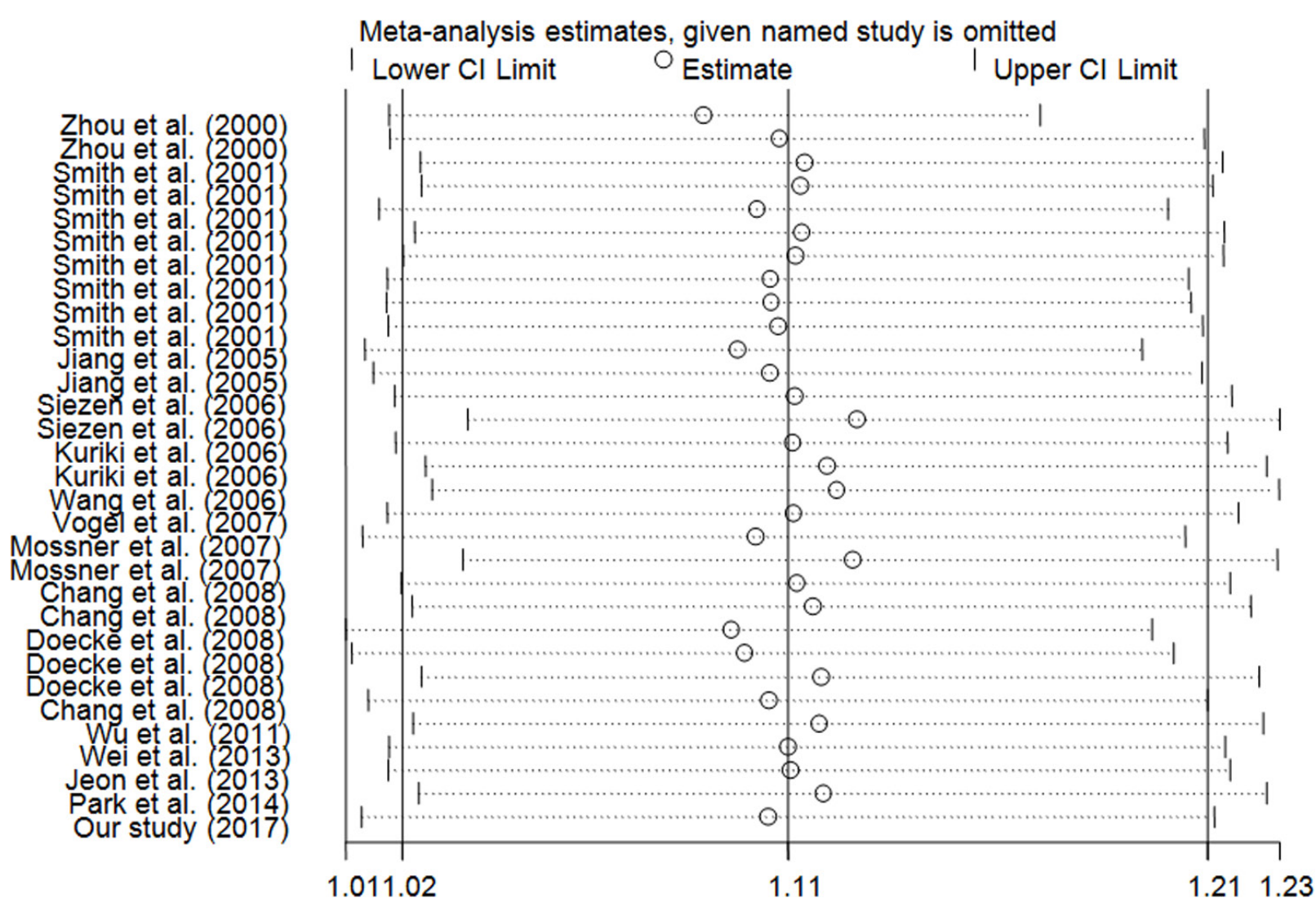

Figure 4: Sensitivity analysis of the influence of TT/CT vs. CC comparison (random-effects estimates for $P P A R G$ c.1347C $>$ T polymorphism). 


\section{DNA extraction and genotyping}

EDTA anticoagulant vacutainer tube was used to collect blood sample. We used DNA Kit (Promega, Madison, USA) to extract the genomic DNA from the whole blood.

PPARG c.1347C > T polymorphism (NP_005028.4: p.His449His) was analyzed using SNPscan ${ }^{\mathrm{TM}}$ genotyping assay (Genesky Biotechologies Inc., Shanghai, China). The SNP assays were confirmed by re-genotyping sixtytwo $(4 \%)$ randomly selected samples.

\section{Statistical analysis}

The continuous variables (e.g. age, and BMI) are presented as the mean $\pm \mathrm{SD}$. We used Student's $t$-test to examine the difference of continuous variables between NSCLC patients and non-cancer controls. In addition, we used $\chi^{2}$ test to determine the difference of categorical variables (e.g. genotypes, smoking status, alcohol consumption, sex, age and BMI). HWE test in controls was undertaken using an internet-based $\chi^{2}$ goodness-of-fit test (http://ihg.gsf.de/cgi-bin/hw/hwa1.pl). Genotype-specific ORs with their corresponding $95 \% \mathrm{CIs}$ and $P$-values were calculated by SAS 9.4 software for windows (SAS Institute, Cary, NC). $P$-values were presented using twosided $\chi^{2}$-test.

\section{Meta-analysis}

To further determine the relationship between $P P A R G$ c. $1347 \mathrm{C}>\mathrm{T}$ variants and cancer susceptibility, we carried out a meta-analysis. All studies focusing on the association between this polymorphism and cancer risk were collected by searching of PubMed and Embase databases (the last search update on June 12, 2017). The search was performed with the terms of (Peroxisome proliferator activated receptor gamma or PPARG) and (NP_005028.4: p.His449His or His449His or $\mathrm{H} 449 \mathrm{H}$ or $\bar{C} 161 \mathrm{~T}$ or $\mathrm{C} 1431 \mathrm{~T}$ or rs 3856806 or c. $1347 \mathrm{C}>\mathrm{T}$ ) and (polymorphism or variant) and (cancer or carcinoma). Additional studies were also supplemented by a hand search of the corresponding references in retrieved articles. In this study, the language of publication was restricted to English. In our analysis, eligible studies had to meet the inclusion criteria: (1) focusing on the association between PPARG c. $1347 \mathrm{C}>\mathrm{T}$ polymorphism and cancer risk; (2) designed as a case-control or cohort study; (3) data could be extracted from the publications (genotypes of cases and controls); (4) published in English language; (5) genotype distribution was consistent with HWE in controls. Two authors (H. Ding and H. Qiu) extracted the detailed information from the eligible publications independently. When they met the disagreement, the third reviewer (Y. Chen) was invited to discuss every item. Finally, a consensus was reached. The following characteristics were selected and collected: the first author, year, country, ethnicity, genotyping method, cancer type, sample size, the origin of cancer cell and genotype frequencies.

For each included study, we analyzed HWE in controls using goodness-of-fit test mentioned above and $P<0.05$ was defined as violation of HWE. Crude ORs with their $95 \%$ CIs were used to examine the strength of relationship between $P P A R G$ c. $1347 \mathrm{C}>\mathrm{T}$ polymorphism and cancer susceptibility. The pooled ORs for this polymorphism were performed under four genetic models (e.g. $\mathrm{TT}+\mathrm{CT}$ vs. $\mathrm{CC}, \mathrm{TT}$ vs. $\mathrm{CC}+\mathrm{CT}$, $\mathrm{TT}$ vs. $\mathrm{CC}$ and $\mathrm{T}$ vs. C). Stratified analyses were extensively performed with respect to origin of cancer cell, ethnicity, cancer type and quality scores. The heterogeneity across the eligible studies was tested by using a $\chi^{2}$-based Q-test and $I^{2}$ test [54]. The pooled OR was calculated by a randomeffects model (the Der-Simonian and Laird method) if $I^{2}>50 \%$ or $P<0.1$, which indicated that heterogeneity was significant $[55,56]$. Otherwise, the pooled OR was assessed by a fixed-effects model (the Mantel-Haenszel method) [57]. Removing each study in turn, sensitivity analysis was carried out by one-way method to determine the stability of the results. Additionally, Begg's test and Egger's linear regression test were conducted to assess the potential publication bias [58] and $P<0.1$ was regarded as a bias. Meta-regression was conducted to analyze the source of heterogeneity [59]. In the present meta-analysis, all statistical analyses were performed by using the STATA 12.0 software for windows (Stata Corporation, College Station, Texas). A $P$ value (two-sided) less than 0.05 were considered significant. Newcastle-Ottawa Quality Assessment Scale was harnessed to determine the quality score of the enrolled studies. If scores $\geq 7$ stars, the study was defined as high-quality $[43,60]$.

\section{ACKNOWLEDGMENTS}

We appreciate all subjects who participated in this study.We wish to thank Dr. Yan Liu (Genesky Biotechnologies Inc., Shanghai, China) for technical support.

\section{CONFLICTS OF INTEREST}

The authors have no potential financial conflicts of interest.

\section{GRANT SUPPORT}

This study was supported in part by Natural Science Foundation of Universities and Colleges of Jiangsu Province (Grant No. 16KJB310002), Senior Talents Scientific Research Foundation of Jiangsu University (Grant No. 16JDG066), Young and Middle-aged Talent Training Project of Health Development Planning Commission in Fujian Province (2016-ZQN-25 and 2014-ZQN-JC-11), Medical Innovation Project of Fujian 
Province (2014-CX-15 and 2014-CX-18), Nursery Garden Project of Fujian Medical University (2015MP020) and Science and Technology Project of Fujian Province (2060203).

\section{REFERENCES}

1. Torre LA, Bray F, Siegel RL, Ferlay J, Lortet-Tieulent J, Jemal A. Global cancer statistics, 2012. CA Cancer J Clin. 2015; 65:87-108

2. Jemal A, Bray F, Center MM, Ferlay J, Ward E, Forman D. Global cancer statistics. CA Cancer J Clin. 2011; 61:69-90.

3. Cai M, Dai S, Chen W, Xia C, Lu L, Dai S, Qi J, Wang M, Wang M, Zhou L, Lei F, Zuo T, Zeng H, et al. Environmental factors, seven GWAS-identified susceptibility loci, and risk of gastric cancer and its precursors in a Chinese population. Cancer Med-US. 2017; 6:708-720.

4. Medina-Gomez G, Gray S, Vidal-Puig A. Adipogenesis and lipotoxicity: role of peroxisome proliferator-activated receptor gamma (PPARgamma), PPARgammacoactivator-1 (PGC1). Public Health Nutr. 2007; 10:1132-1137.

5. Gandhi GR, Stalin A, Balakrishna K, Ignacimuthu S, Paulraj MG, Vishal R. Insulin sensitization via partial agonism of PPARgamma and glucose uptake through translocation and activation of GLUT4 in PI3K/p-Akt signaling pathway by embelin in type 2 diabetic rats. Biochim Biophys Acta. 2013; 1830:2243-2255.

6. Fucci A, Colangelo T, Votino C, Pancione M, Sabatino L, Colantuoni V. The role of peroxisome proliferator-activated receptors in the esophageal, gastric, and colorectal cancer. PPAR Res. 2012; 2012:242498.

7. Robbins GT, Nie D. PPAR gamma, bioactive lipids, and cancer progression. Front Biosci (Landmark Ed). 2012; 17:1816-1834.

8. Deeb SS, Fajas L, Nemoto M, Pihlajamaki J, Mykkanen L, Kuusisto J, Laakso M, Fujimoto W, Auwerx J. A Pro12Ala substitution in PPARgamma2 associated with decreased receptor activity, lower body mass index and improved insulin sensitivity. Nat Genet. 1998; 20:284-287.

9. Hutter S, Knabl J, Andergassen U, Jeschke U. The Role of PPARs in Placental Immunology: A Systematic Review of the Literature. PPAR Res. 2013; 2013:970276.

10. Mantovani A, Allavena P, Sica A, Balkwill F. Cancer-related inflammation. Nature. 2008; 454:436-444.

11. Khandekar MJ, Cohen P, Spiegelman BM. Molecular mechanisms of cancer development in obesity. Nat Rev Cancer. 2011; 11:886-895.

12. Calle EE, Kaaks R. Overweight, obesity and cancer: epidemiological evidence and proposed mechanisms. Nat Rev Cancer. 2004; 4:579-591.

13. Crous-Bou M, Rennert G, Salazar R, Rodriguez-Moranta F, Rennert HS, Lejbkowicz F, Kopelovich L, Lipkin SM, Gruber SB, Moreno V. Genetic polymorphisms in fatty acid metabolism genes and colorectal cancer. Mutagenesis. 2012; 27:169-176.

14. Abuli A, Fernandez-Rozadilla C, Alonso-Espinaco V, Munoz J, Gonzalo V, Bessa X, Gonzalez D, Clofent J, Cubiella J, Morillas JD, Rigau J, Latorre M, FernandezBanares F, et al. Case-control study for colorectal cancer genetic susceptibility in EPICOLON: previously identified variants and mucins. BMC cancer. 2011; 11:339.

15. Tsilidis KK, Helzlsouer KJ, Smith MW, Grinberg V, Hoffman-Bolton J, Clipp SL, Visvanathan K, Platz EA. Association of common polymorphisms in IL10, and in other genes related to inflammatory response and obesity with colorectal cancer. Cancer Causes Control. 2009; 20:1739-1751.

16. Kury S, Buecher B, Robiou-du-Pont S, Scoul C, Colman H, Le Neel T, Le Houerou C, Faroux R, Ollivry J, Lafraise B, Chupin LD, Sebille V, Bezieau S. Low-penetrance alleles predisposing to sporadic colorectal cancers: a French casecontrolled genetic association study. BMC cancer. 2008; $8: 326$.

17. Kuriki K, Hirose $\mathrm{K}$, Matsuo $\mathrm{K}$, Wakai K, Ito $\mathrm{H}$, Kanemitsu Y, Hirai T, Kato T, Hamajima N, Takezaki T, Suzuki T, Saito T, Tanaka R, et al. Meat, milk, saturated fatty acids, the Pro12Ala and C161T polymorphisms of the PPARgamma gene and colorectal cancer risk in Japanese. Cancer Sci. 2006; 97:1226-1235.

18. Theodoropoulos G, Papaconstantinou I, Felekouras E, Nikiteas N, Karakitsos P, Panoussopoulos D, Lazaris A, Patsouris E, Bramis J, Gazouli M. Relation between common polymorphisms in genes related to inflammatory response and colorectal cancer. World J Gastroenterol. 2006; 12:5037-5043.

19. Slattery ML, Curtin K, Wolff R, Ma KN, Sweeney C, Murtaugh M, Potter JD, Levin TR, Samowitz W. PPARgamma and colon and rectal cancer: associations with specific tumor mutations, aspirin, ibuprofen and insulinrelated genes (United States). Cancer Causes Control. 2006; 17:239-249.

20. McGreavey LE, Turner F, Smith G, Boylan K, Timothy Bishop D, Forman D, Roland Wolf C, Barrett JH, Colorectal Cancer Study G. No evidence that polymorphisms in CYP2C8, CYP2C9, UGT1A6, PPARdelta and PPARgamma act as modifiers of the protective effect of regular NSAID use on the risk of colorectal carcinoma. Pharmacogenet Genomics. 2005; 15:713-721.

21. Jiang J, Gajalakshmi V, Wang J, Kuriki K, Suzuki S, Nakamura S, Akasaka S, Ishikawa H, Tokudome S. Influence of the C161T but not Pro12Ala polymorphism in the peroxisome proliferator-activated receptor-gamma on colorectal cancer in an Indian population. Cancer Sci. 2005; 96:507-512.

22. Landi S, Moreno V, Gioia-Patricola L, Guino E, Navarro M, de Oca J, Capella G, Canzian F, Bellvitge Colorectal Cancer Study G. Association of common polymorphisms in 
inflammatory genes interleukin (IL)6, IL8, tumor necrosis factor alpha, NFKB1, and peroxisome proliferator-activated receptor gamma with colorectal cancer. Cancer Res. 2003; 63:3560-3566.

23. Vogel U, Christensen J, Dybdahl M, Friis S, Hansen RD, Wallin H, Nexo BA, Raaschou-Nielsen O, Andersen PS, Overvad K, Tjonneland A. Prospective study of interaction between alcohol, NSAID use and polymorphisms in genes involved in the inflammatory response in relation to risk of colorectal cancer. Mutat Res. 2007; 624:88-100.

24. Siezen CL, Bueno-de-Mesquita HB, Peeters PH, Kram NR, van Doeselaar M, van Kranen HJ. Polymorphisms in the genes involved in the arachidonic acid-pathway, fish consumption and the risk of colorectal cancer. Int J Cancer. 2006; 119:297-303.

25. Lim WY, Chen Y, Ali SM, Chuah KL, Eng P, Leong SS, Lim E, Lim TK, Ng AW, Poh WT, Tee A, Teh M, Salim A, et al. Polymorphisms in inflammatory pathway genes, host factors and lung cancer risk in Chinese female neversmokers. Carcinogenesis. 2011; 32:522-529.

26. Campa D, Zienolddiny S, Maggini V, Skaug V, Haugen A, Canzian F. Association of a common polymorphism in the cyclooxygenase 2 gene with risk of non-small cell lung cancer. Carcinogenesis. 2004; 25:229-235.

27. Vogel U, Christensen J, Wallin H, Friis S, Nexo BA, Raaschou-Nielsen O, Overvad K, Tjonneland A. Polymorphisms in genes involved in the inflammatory response and interaction with NSAID use or smoking in relation to lung cancer risk in a prospective study. Mutat Res. 2008; 639:89-100.

28. Zhang YT, Mao W. The Pro12Ala polymorphism of peroxisome proliferator - activated receptor - gamma 2 gene in lung cancer. Zhejiang Med. 2007; 29:1257-1259.

29. Wang Y, Chen Y, Jiang H, Tang W, Kang M, Liu T, Guo Z, Ma Z. Peroxisome proliferator-activated receptor gamma (PPARG) rs1801282 C $>\mathrm{G}$ polymorphism is associated with cancer susceptibility in asians: an updated meta-analysis. Int J Clin Exp Med. 2015; 8:12661-12673.

30. Tang W, Chen Y, Wang Y, Gu H, Chen S, Kang M. Peroxisome proliferator-activated receptor gamma (PPARG) polymorphisms and breast cancer susceptibility: a meta-analysis. Int J Clin Exp Med. 2015; 8:12226-12238.

31. Xu W, Li Y, Wang X, Chen B, Liu S, Wang Y, Zhao W, Wu J. PPARgamma polymorphisms and cancer risk: a meta-analysis involving 32,138 subjects. Oncol Rep. 2010; 24:579-585.

32. Pan HF, Leng RX, Ye DQ. Lack of association of interleukin-18 gene promoter $-607 \mathrm{~A} / \mathrm{C}$ polymorphism with susceptibility to autoimmune diseases: a meta-analysis. Lupus. 2011; 20:945-951.

33. Chang SC, Rashid A, Gao YT, Andreotti G, Shen MC, Wang BS, Han TQ, Zhang BH, Sakoda LC, Leitzmann MF, Chen BE, Rosenberg PS, Chen J, et al. Polymorphism of genes related to insulin sensitivity and the risk of biliary tract cancer and biliary stone: a population-based casecontrol study in Shanghai, China. Carcinogenesis. 2008; 29:944-948.

34. Smith WM, Zhou XP, Kurose K, Gao X, Latif F, Kroll T, Sugano K, Cannistra SA, Clinton SK, Maher ER, Prior TW, Eng C. Opposite association of two PPARG variants with cancer: overrepresentation of $\mathrm{H} 449 \mathrm{H}$ in endometrial carcinoma cases and underrepresentation of P12A in renal cell carcinoma cases. Hum Genet. 2001; 109:146-151.

35. Wu MH, Chu CH, Chou YC, Chou WY, Yang T, Hsu GC, $\mathrm{Yu} \mathrm{CP}$, Yu JC, Sun CA. Joint effect of peroxisome proliferator-activated receptor gamma genetic polymorphisms and estrogen-related risk factors on breast cancer risk: results from a case-control study in Taiwan. Breast Cancer Res Treat. 2011; 127:777-784.

36. Park B, Shin A, Kim KZ, Lee YS, Hwang JA, Kim Y, Sung J, Yoo KY, Lee ES. Lack of effects of peroxisome proliferatoractivated receptor gamma genetic polymorphisms on breast cancer risk: a case-control study and pooled analysis. Asian Pac J Cancer Prev. 2014; 15:9093-9099.

37. Wei W, Jiang M, Luo L, Li Z, Wang P, Dong WQ. Colorectal cancer susceptibility variants alter risk of breast cancer in a Chinese Han population. Genet Mol Res. 2013; 12:6268-6274.

38. Doecke JD, Zhao ZZ, Stark MS, Green AC, Hayward NK, Montgomery GW, Webb PM, Whiteman DC, Australian Cancer S. Single nucleotide polymorphisms in obesityrelated genes and the risk of esophageal cancers. Cancer Epidemiol Biomarkers Prev. 2008; 17:1007-1012.

39. Jeon C, Chang SC, Mu L, Zhao J, Rao JY, Lu QY, Zhang ZF. Genetic variants of peroxisome proliferatoractivated receptor delta are associated with gastric cancer. Dig Dis Sci. 2013; 58:2881-2886.

40. Zhou XP, Smith WM, Gimm O, Mueller E, Gao X, Sarraf P, Prior TW, Plass C, von Deimling A, Black PM, Yates AJ, Eng C. Over-representation of PPARgamma sequence variants in sporadic cases of glioblastoma multiforme: preliminary evidence for common low penetrance modifiers for brain tumour risk in the general population. J Med Genet. 2000; 37:410-414.

41. Wang SS, Davis S, Cerhan JR, Hartge P, Severson RK, Cozen W, Lan Q, Welch R, Chanock SJ, Rothman N. Polymorphisms in oxidative stress genes and risk for nonHodgkin lymphoma. Carcinogenesis. 2006; 27:1828-1834.

42. Mossner R, Meyer P, Jankowski F, Konig IR, Kruger U, Kammerer S, Westphal G, Boettger MB, Berking C, Schmitt C, Brockmoller J, Ziegler A, Stapelmann H, et al. Variations in the peroxisome proliferator-activated receptor-gamma gene and melanoma risk. Cancer Lett. 2007; 246:218-223.

43. Wang W, Shao Y, Tang S, Cheng X, Lian H, Qin C. Peroxisome proliferator-activated receptor-gamma (PPARgamma) Pro12Ala polymorphism and colorectal cancer (CRC) risk. Int J Clin Exp Med. 2015; 8:4066-4072. 
44. Lecarpentier Y, Claes V, Vallee A, Hebert JL. Interactions between PPAR Gamma and the Canonical Wnt/BetaCatenin Pathway in Type 2 Diabetes and Colon Cancer. PPAR Res. 2017; 2017:5879090.

45. Lagana AS, Vitale SG, Nigro A, Sofo V, Salmeri FM, Rossetti P, Rapisarda AM, La Vignera S, Condorelli RA, Rizzo G, Buscema M. Pleiotropic Actions of Peroxisome Proliferator-Activated Receptors (PPARs) in Dysregulated Metabolic Homeostasis, Inflammation, Cancer: Current Evidence and Future Perspectives. Int J Mol Sci. 2016; 17.

46. Ivanova EA, Myasoedova VA, Melnichenko AA, Orekhov AN. Peroxisome Proliferator-Activated Receptor (PPAR) Gamma Agonists as Therapeutic Agents for Cardiovascular Disorders: Focus on Atherosclerosis. Curr Pharm Des. 2017; 23:1119-1124.

47. Yu J, Qiu Y, Yang J, Bian S, Chen G, Deng M, Kang H, Huang L. DNMT1-PPARgamma pathway in macrophages regulates chronic inflammation and atherosclerosis development in mice. Sci Rep. 2016; 6:30053.

48. Brower V. Illuminating the diabetes-cancer link. J Natl Cancer Inst. 2012; 104:1048-1050.

49. Wang D, Dubois RN. Associations between obesity and cancer: the role of fatty acid synthase. J Natl Cancer Inst. 2012; 104:343-345.

50. Lv X, Zhang L, Sun J, Cai Z, Gu Q, Zhang R, Shan A. Interaction between peroxisome proliferator-activated receptor gamma polymorphism and obesity on type 2 diabetes in a Chinese Han population. Diabetol Metab Syndr. 2017; 9:7.

51. Lu Y, Ye X, Cao Y, Li Q, Yu X, Cheng J, Gao Y, Ma J, $\mathrm{Du} \mathrm{W}$, Zhou L. Genetic variants in peroxisome proliferatoractivated receptor-gamma and retinoid $\mathrm{X}$ receptor-alpha gene and type 2 diabetes risk: a case-control study of a Chinese Han population. Diabetes Technol Ther. 2011; 13:157-164.
52. Du J, Shi H, Lu Y, Du W, Cao Y, Li Q, Ma J, Ye X, Cheng J, Yu X, Gao Y, Zhou L. Tagging single nucleotide polymorphisms in the PPAR-gamma and RXR-alpha gene and type 2 diabetes risk: a case-control study of a Chinese Han population. J Biomed Res. 2011; 25:33-41.

53. Wang P, Wang Q, Yin Y, Yang Z, Li W, Liang D, Zhou P. Association between Peroxisome Proliferator-activated Receptor Gamma Gene Polymorphisms and Atherosclerotic Diseases: A Meta-analysis of Case-control Studies. J Atheroscler Thromb. 2015; 22:912-925.

54. Higgins JP, Thompson SG. Quantifying heterogeneity in a meta-analysis. Stat Med. 2002; 21:1539-1558.

55. Higgins JP, Thompson SG, Deeks JJ, Altman DG. Measuring inconsistency in meta-analyses. Bmj. 2003; 327:557-560.

56. DerSimonian R, Laird N. Meta-analysis in clinical trials. Control Clin Trials. 1986; 7:177-188.

57. Mantel N, Haenszel W. Statistical aspects of the analysis of data from retrospective studies of disease. J Natl Cancer Inst. 1959; 22:719-748.

58. Egger M, Davey Smith G, Schneider M, Minder C. Bias in meta-analysis detected by a simple, graphical test. Bmj. 1997; 315:629-634.

59. Whitehead A, Whitehead J. A general parametric approach to the meta-analysis of randomized clinical trials. Stat Med. 1991; 10:1665-1677.

60. Wang Y, Huang Q, Liu J, Wang Y, Zheng G, Lin L, Yu H, Tang W, Huang Z. Vascular endothelial growth factor A polymorphisms are associated with increased risk of coronary heart disease: a meta-analysis. Oncotarget. 2017; 8:30539-30551. https://doi.org/10.18632/oncotarget.15546. 